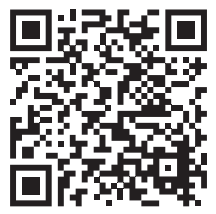

\title{
Manifestación ocular atípica en síndrome de activación de macrófago que complica la artritis idiopática juvenil sistémica
}

\author{
Miguel García-Domínguez, ${ }^{*}$ Eduardo González-López, ${ }^{\ddagger}$ \\ Daniela Arce-Cabrera, ${ }^{\S}$ Rosalía Heras-Salazarll
}

\begin{abstract}
RESUMEN
El síndrome de activación de macrófago que complica la artritis idiopática juvenil de inicio sistémico es una condición grave y potencialmente fatal. Se caracteriza por hiperinflamación aguda con fiebre, citopenias, disfunción multiorgánica y evidencia de hemofagocitosis en el sistema reticuloendotelial con actividad incrementada del macrófago. Presentamos el caso de un adolescente de 13 años de edad con síndrome de activación de macrófago con artritis idiopática juvenil de inicio sistémico que desarrolló retinocoroiditis infiltrativa bilateral con componente vascular isquémico y compromiso grave de la visión.
\end{abstract}

Palabras clave: Síndrome de activación de macrófago, linfohistiocitosis hemofagocítica, artritis idiopática juvenil sistémica, coroiditis, retinitis.

\begin{abstract}
Macrophage activation syndrome complicating systemic-onset juvenile idiopathic arthritis is a serious and potentially life-threatening condition. Characterized of acute hyperinflammation with fever, cytopenias, multiple organ dysfunction, and hemophagocytosis in the reticuloendothelial system with increased macrophage activity. We present adolescent of a 13-year-old with systemic-onset juvenile idiopathic arthritis complicated by macrophage activation syndrome, who developed bilateral infiltrative retinochoroiditis with an ischemic vascular component and severe vision impairment.
\end{abstract}

Keywords: Macrophage activation syndrome, hemophagocytic lymphohistiocytosis, systemic juvenile idiopathic arthritis, choroiditis, retinitis.

\section{INTRODUCCIÓN}

El síndrome de activación del macrófago (SAM) es una forma reactiva de linfohistiocitosis hemofagocítica (HLH) de presentación aguda, grave y potencialmente fatal, debido a una activación inapropiada de macrófagos y citocinas proinflamatorias que se presentan como complicación de enfermedades autoinmunes, como artritis idiopática juvenil de inicio sistémico (AIJs), enfermedad de Still en adultos; sin embargo, puede observarse en

\footnotetext{
* Departamento de Inmunología y Alergia.

* Departamento de Pediatría Médica.

\& Departamento de Oncología Pediátrica.

" Departamento de Oftalmología Pediátrica.
}

Hospital Pediátrico de Sinaloa. Culiacán, Sinaloa, México. 
Vol. 29, Núm. $2 \cdot$ Mayo-Agosto 2020

Tabla 1: Estudios de laboratorio y gabinete.

Laboratorios de rutina

Microbiológicos (negativos)

Inmunológicos (negativos)

Aspirado MO

Imagenología

Tratamiento
$\mathrm{Hb} 10.4 \mathrm{~g} / \mathrm{dL}$, Hto $30.5 \%$, leucocitos 3,480/ $\mathrm{mm}^{3}$, neutrófilos $2,630 / \mathrm{mm}^{3}$, linfocitos $480 / \mathrm{mm}^{3}$, plaquetas $19,000 / \mathrm{mm}^{3}$, PCR $10 \mathrm{mg} / \mathrm{dL}$, VSG $25 \mathrm{~mm} / \mathrm{h}$, PCT $15.74 \mathrm{ng} / \mathrm{mL}$, creatinina $0.65 \mathrm{mg} / \mathrm{dL}, \mathrm{DHL} 1567 \mathrm{UI} / \mathrm{L}$, bilirrubina total $0.65 \mathrm{mg} / \mathrm{dL}$, bilirrubina directa $0.33 \mathrm{mg} / \mathrm{dL}$, bilirrubina indirecta $0.32 \mathrm{mg} / \mathrm{dL}$, AST $202 \mathrm{UI} / \mathrm{L}$, ALT $73 \mathrm{UI} / \mathrm{L}$, albúmina $2.6 \mathrm{~g} / \mathrm{dL}$, TP 15.4 (62\% de actividad), INR 1.22, TTPa 36, FA 293 UI/L, colesterol $108 \mathrm{mg} / \mathrm{dL}$, TGR $576 \mathrm{mg} / \mathrm{dL}$, fibrinógeno $106 \mathrm{mg} / \mathrm{dL}$, ferritina $1,500 \mathrm{ng} / \mathrm{mL}$

Dengue IgM, leptospira IgM, hemocultivos, perfil TORCH, VIH, BAAR, PPD, VHS 1 y 2, brucelosis, serología para VEB

LCR: trasparente, leucocitos 8 mm³, PMN 37\%, MN 63\%, glucosa: 70 mg/dL, proteínas: 61 mg/L, Frote Gram (-)

Ac anticardiolipinas $\lg \mathrm{G}$ e $\lg \mathrm{M}$, anticoagulante lúpico, Ac, antibeta 2 glicoproteína $\lg \mathrm{G}$ e $\lg \mathrm{M}, \mathrm{Ac}$ antiperoxidasa, $\mathrm{p}$ y $\mathrm{c}$ ANCA, ANA, Ac anti DNA nativo, Coombs directo

Hipercelularidad con hemofagocitosis

USG: bazo de 12.7 x 8.2 cm e hígado en todos sus diámetros, lodo biliar, líquido libre en cavidad $200 \mathrm{~cm}^{3}$ aproximadamente TAC de cráneo simple y contrastado: sin evidencia de alteraciones estructurales

Cefotaxima $150 \mathrm{mg} / \mathrm{kg} / \mathrm{día}$, ciprofloxacino $30 \mathrm{mg} / \mathrm{kg} / \mathrm{día}$, fluconazol $6 \mathrm{mg} / \mathrm{kg} / \mathrm{día}$

Dexametasona $10 \mathrm{mg} / \mathrm{m}^{2} \mathrm{SC}$, ciclosporina $2.5 \mathrm{mg} / \mathrm{kg} /$ día, metotrexate $15 \mathrm{mg} / \mathrm{m}^{2} \mathrm{SC} / \mathrm{sem}$, IGIV $1 \mathrm{~g} / \mathrm{kg} / \mathrm{do}$, metilprednisolona (30 mg/kg/do)

ANCA = anticuerpos contra el citoplasma del neutrófilo; IGIV = inmunoglobulina intravenosa; LCR = líquido cefalorraquídeo; $A N A=$ anticuerpos antinucleares.

lupus eritematoso sistémico (LES) y enfermedad de Kawasaki (EK), entre otros. ${ }^{1,2}$

En la etiología se consideran aspectos genéticos en las HLH familiares, mientras que en las formas secundarias, los procesos infecciosos (VEB, CMV, etcétera), linfoproliferativos o autoinmunes. EI SAM en las enfermedades autoinmunes puede ser desencadenado por infecciones, fármacos y actividad de la enfermedad autoinmune con la consecuente liberación de citocinas como: interleucina 1 (IL-1), IL-6, IL-18, factor de necrosis tumoral alfa (TNF- $\alpha)$, interferón gamma (INF- $\gamma$ ), entre otros. ${ }^{3}$

Las manifestaciones clínicas abarcan: fiebre de alto grado, hepatomegalia, esplenomegalia, citopenias, coagulopatía e hiperinflamación con reactantes de fase aguda incrementados, altos niveles de ferritina sérica, así como actividad incrementada del macrófago con hemofagocitosis en tejidos que conforman el sistema reticuloendotelial, como se determina en el aspirado de médula ósea (MO). Se han establecido criterios diagnósticos para HLH por la Sociedad del Histiocito 2004, ${ }^{4}$ para trastornos genéticos homocigóticos que conducen a hemofagocitosis y que no son necesariamente oportunos para SAM en el entorno de las enfermedades autoinmunes. EI SAM que se presenta en las enfermedades autoinmunes muestra características diferentes, como: hiperferritinemia marcada, descenso en la velocidad de sedimentación globular (VSG), citopenias poco marcadas y coagulopatía acentuada al inicio de los síntomas. ${ }^{5}$

El tratamiento debe instaurarse de forma inmediata incluyendo la eliminación de posibles desencadenantes, así como la supresión de la respuesta inflamatoria con dosis altas de esteroides y ciclosporina $A$, menos frecuente el etopósido y biológicos (IL-1). ${ }^{6}$
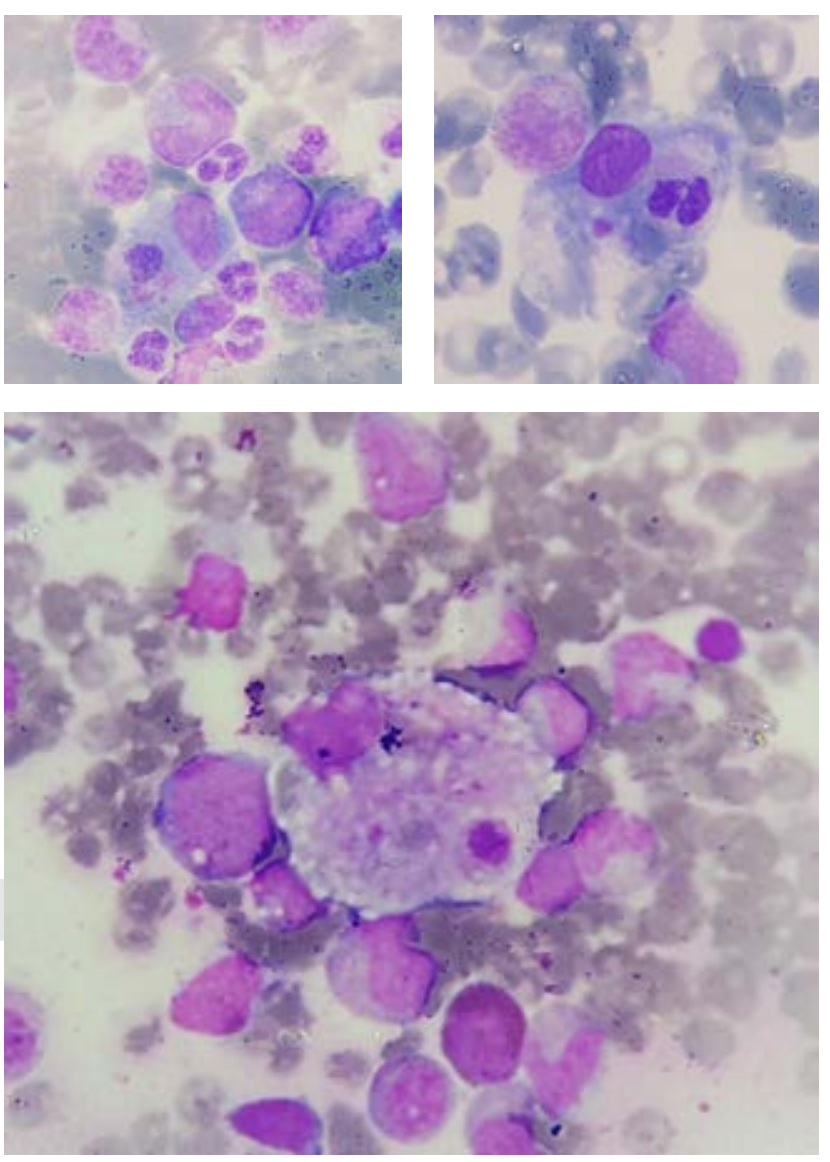

Figura 1: Aspirado de médula ósea: se observan células en diferentes estadios de maduración de la serie blanca, eritroblastos normales, sin células inmaduras, con macrófagos fagocitando eritrocitos y leucocitos en su citoplasma. 
Vol. 29, Núm. 2 • Mayo-Agosto 2020

La evolución, pronóstico y mortalidad están condicionados por el tiempo de retraso en el diagnóstico, así como el factor que motivó el SAM. Las manifestaciones oculares en las enfermedades autoinmunes pueden estar presentes al diagnóstico o evolución; sin embargo, en síndrome hemofagocítico son hallazgos inusuales y en su mayoría están asociados a infección viral. El objetivo de este manuscrito es describir los hallazgos oculares inusuales y atípicas en un paciente con SAM secundario a AlJs.

\section{CASO CLÍNICO}

Masculino de 13 años, sin antecedentes de importancia. Inicia cinco días previos con fiebre de $40{ }^{\circ} \mathrm{C}$ ( 3 a 4 episodios/día) sin predominio de horario, astenia, adinamia, mioartralgias, cefalea frontal, dolor retro-ocular bilateral e hiporexia. A pesar de manejo sintomático mantuvo persistencia de la fiebre, lo que motivó aplicación de penicilina benzatínica intramuscular. Desarrolló eritema urticariforme en tronco y extremidades. Fue referido al hospital con serología para dengue negativa y hallazgo de trombocitopenia de $19,000 / \mathrm{mm}^{3}$.

A su ingreso hospitalario persistía febril, con datos de hipoperfusión tisular y choque, instaurando reanimación hídrica y aminas (norepinefrina-dobutamina), así como cefotaxima $150 \mathrm{mg} / \mathrm{kg} / \mathrm{día}$. Con presencia de exantema generalizado, adenopatías cervicales e inguinales de $7 \times 10 \mathrm{~mm}$, esplenomegalia de $3 \mathrm{~cm}$ bajo el borde costal, anemia, trombocitopenia, hipofibrinogenemia e hiperferritinemia (Tabla 1), obligó a realizar aspirado de médula ósea (MO) confirmando síndrome hemofagocítico (Figura 1).

Se inició protocolo HLH-04 con dexametasona (10 $\mathrm{mg} / \mathrm{m}^{2} \mathrm{SC}$ ) y etopósido $\left(150 \mathrm{mg} / \mathrm{m}^{2} \mathrm{SC}\right)$ presentando me- joría clínica y bioquímica a las 72 horas. Al quinto día nuevamente fiebre de alto grado cambiando esquema a ciprofloxacino (30 mg/kg/día) y fluconazol (6 mg/kg/día). Cultivos y hemocultivos negativos, perfil TORCH, VEB, $\mathrm{VIH}, \mathrm{VHC}$ y VHB negativos, se realizó nuevo aspirado de $\mathrm{MO}$ al octavo día sin evidenciar hemofagocitosis. Al noveno día presentó súbitamente disminución de la agudeza visual: ojo derecho (OD) 20/250 y ojo izquierdo (OI) 20/160. El fondo de ojo demostró exudados blandos subretinianos en la periferia del nervio óptico y polo posterior, zonas con microhemorragias y microinfartos en arcada temporal inferior (Figura 2). Se administró inmunoglobulina intravenosa (IVIG) $1 \mathrm{~g} / \mathrm{kg} /$ dosis y bolo de metilprednisolona (30 mg/kg/dosis) tres días continuos debido al compromiso ocular, inflamación sistémica y sin evidencia serológica de algún microorganismo. En retrospectiva con los hallazgos oculares sugestivos de vasculitis (retinocoroiditis infiltrativa con isquemia vascular central bilateral), se determinó la etiología y criterios para AlJs: fiebre de alto grado, rash evanescente, linfadenopatía generalizada, hepatoesplenomegalia con exclusión de otras causas posibles.

La evolución sistémica fue favorable: ausencia de fiebre, normalización de ferritina, VSG, PCR y mejora parcial de la agudeza visual. La fluorangiografía ocular mostró atrofia en área macular secundaria al cuadro de retinocoroiditis (Figura 3). Se egresó al día 25 con protocolo HLH-04, ciclosporina $2.5 \mathrm{mg} / \mathrm{kg} /$ día, dexametasona $5 \mathrm{mg} / \mathrm{m}^{2} \mathrm{SC}$ y etopósido $150 \mathrm{mg} / \mathrm{m}^{2} \mathrm{SC} / \mathrm{d}$.

\section{DISCUSIÓN}

El diagnóstico de AlJs es de exclusión y continúa siendo un desafío para el médico de primer contacto. Constituye del $10-15 \%$ de las formas de AIJ, con los siguientes
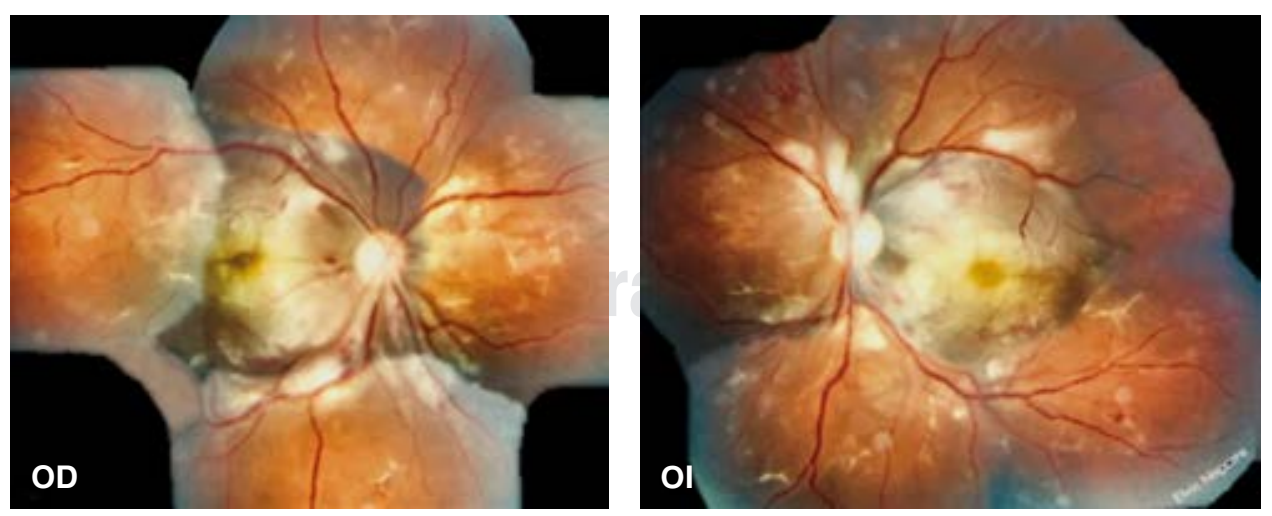

Figura 2: Angiografía con fluoresceína: nervio óptico con bordes nítidos con palidez marcada del lado temporal bilateral. Emergencia de la red vascular con marcada dilatación y tortuosidad de la red venosa con hemorragias en mancha en el has papilar macular, área central de la retina con exudados infiltrativos, discretos exudados duros centrales y de promedio en ojo izquierdo. Palidez de toda la retina central y paracentral por reducción acentuada en la perfusión y por bloqueo exudativo coroideo. Se observan shunts opto-peripapilares con zonas de hipofluorescencia periférica. Llenado arterial periférico no afectado. 
Vol. 29, Núm. 2 • Mayo-Agosto 2020
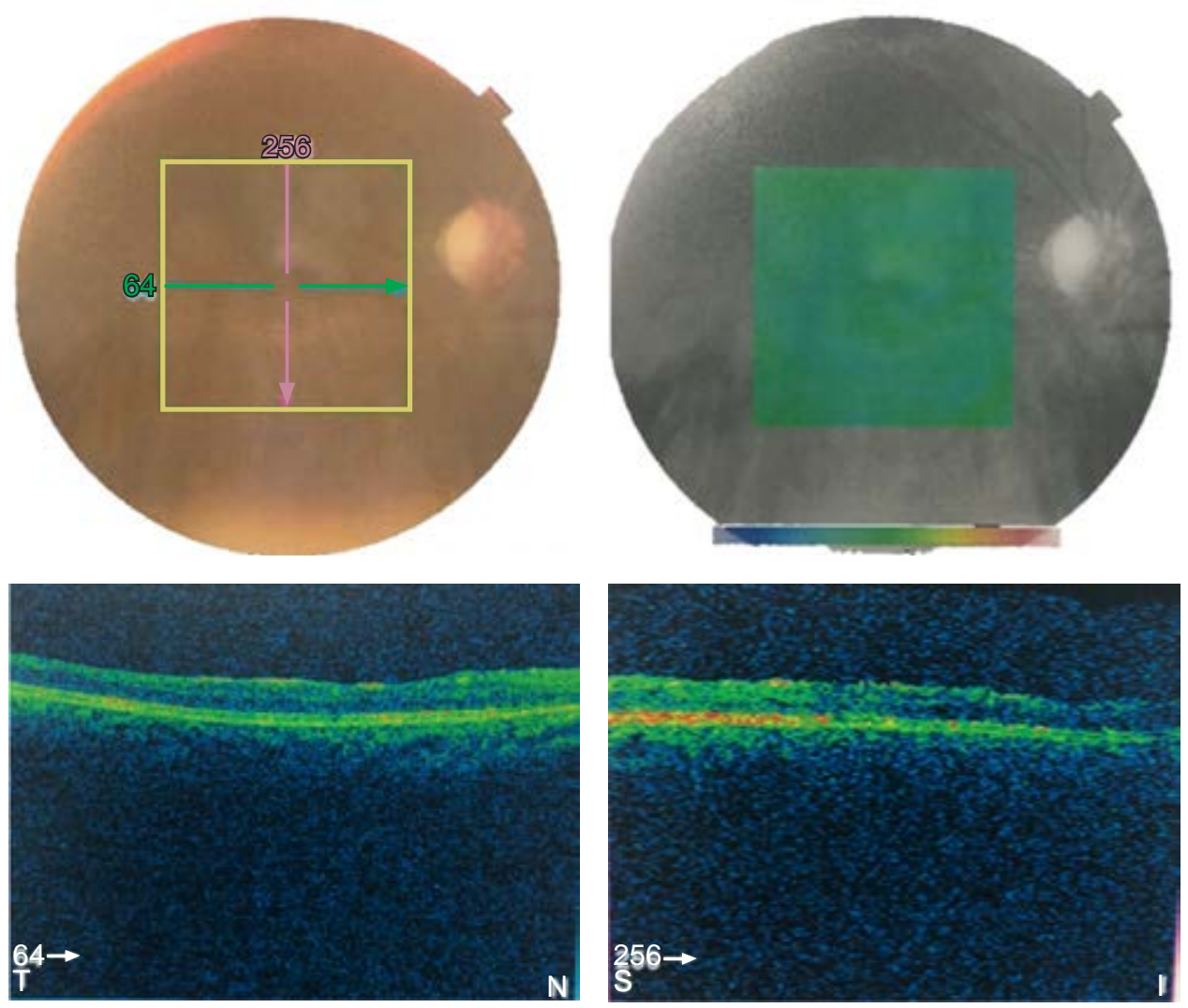

Figura 3:

Tomografía óptica coherente de OD: atrofia macular con grosor de capa de fibras nerviosas de $195.4 \mu \mathrm{m}$, conservando zona de la fóvea un grosor mayor de $206 \mu \mathrm{m}$. No fue posible realizar el mapa de capa de fibras nerviosas del área macular de OI por la incapacidad de fijación visual.

criterios descritos por la Liga Internacional de Asociaciones de Reumatología (ILAR): artritis en una o más articulaciones acompañada o precedida por fiebre de al menos dos semanas de duración, que se documenta a diario durante al menos tres días, acompañado de uno o más de los siguientes hallazgos: 1) exantema eritematoso evanescente; 2) linfadenopatía; 3) hepatomegalia y/o esplenomegalia y, 4) serositis. ${ }^{7}$ EI SAM se define como una forma reactiva de $\mathrm{HLH}$ en enfermedades autoinmunes, que puede presentarse simultáneamente con AlJs o durante el curso de la enfermedad, la cual supone el $10 \%$ de los pacientes y hasta un $30-43 \%$ de forma subclínica, con una elevada mortalidad. ${ }^{6}$ Recientemente
Ravelli y colaboradores emitieron criterios para definir el SAM que complica AIJs (Tabla 2). ${ }^{8}$

La asociación de AlJs y SAM es de alrededor del 7\%, en la población pediátrica se ha documentado con menor frecuencia, seguido de LES, dermatomiositis y/o enfermedad de Kawasaki. ${ }^{9-11}$

Las manifestaciones oculares en AlJ se describen hasta en $30 \%,{ }^{12}$ siendo más frecuente la uveítis e iridocliclitis (20\%), con dos picos de edad; mujeres con AIJ oligoarticular y ANA (+) entre los dos a cuatro años, y varones de 10 a 12 años. ${ }^{13,14}$ Mientras que en las manifestaciones oculares en HLH se reportan casos aislados y asociadas a infecciones virales. Suhr y su equipo ${ }^{15}$

Tabla 2: Criterios de SAM en paciente con AlJs y criterios del paciente.

\begin{tabular}{ll}
\hline Diagnóstico de SAM-AlJs & Paciente \\
\hline $\begin{array}{l}\text { Paciente febril en el que se conoce o sospecha AlJs que presenta una } \\
\text { ferritina }>684 \mathrm{ng} / \mathrm{mL} \text { y dos o más de las siguientes alteraciones }\end{array}$ & Fiebre: 5 días previos, con ferritina: $1500 \mathrm{ng} / \mathrm{mL}$ \\
- Plaquetas: $\leq 181 \times 10^{9} / \mathrm{L}$ & - Plaquetas: $19 \times 10^{9} / \mathrm{L}$ \\
- AST/TGO: $>48 \mathrm{U} / \mathrm{L}$ & - AST: $202 \mathrm{U} / \mathrm{L}$ \\
- Triglicéridos: $>156 \mathrm{mg} / \mathrm{dL}$ & - Triglicéridos: $576 \mathrm{mg} / \mathrm{dL}$ \\
- Fibrinógeno: $\leq 360 \mathrm{mg} / \mathrm{dL}$ & - Fibrinógeno: $106 \mathrm{mg} / \mathrm{dL}$
\end{tabular}

SAM = síndrome de activación de macrófago; AlJs = artritis idiopática juvenil sistémica. 
Vol. 29, Núm. 2・ Mayo-Agosto 2020

reportan nueve casos HLH familiar con los siguientes hallazgos oculares: edema periorbitario, papiledema, opacidades blanquecinas en vítreo, infiltrados perivenosos, derrame coroideo con desprendimiento secundario de retina, así como panuveítis. Cuatro HLH secundarios a infecciones virales: hemorragias en astilla, vasos con leve tortuosidad, lesiones perivasculares blanquecinas, desprendimiento epitelial y edema macular. Vizcaino y su grupo reportan tres adultos post mortem con síndrome hemofagocítico con afección ocular, con infiltrado inflamatorio bilateral confinado a coroides con presencia de macrófagos y escasos linfocitos, infiltrado linfohistiocítico bilateral con afección del cuerpo ciliar de coroides e infiltrado difuso bilateral inflamatorio, con predominio de macrófagos en cuerpo ciliar e iris. ${ }^{16}$

La asociación AlJs-MAS con manifestaciones oculares no está reportada en las diversas series de casos; Ruscitti y colaboradores ${ }^{17}$ reportan 10 pacientes sin determinar la existencia de afección ocular. En el caso de nuestro paciente no hubo aislamiento de los gérmenes involucrados en afección ocular descritos para HLH, pero la evidencia de signos inflamatorios y/o vasculitis ocular determinó en la revisión retrospectiva del caso criterios para AlJs. Actualmente con atrofia macular bilateral (Figura 3).

\section{CONCLUSIONES}

Las manifestaciones oculares son poco frecuentes en el síndrome hematofagocítico, en artritis idiopática juvenil puede presentarse en el curso de la evolución. La detección de vasculitis a nivel ocular obliga a instaurar inmunosupresores e inmunomodulación asociada a tratamiento del síndrome hematofagocítico para evitar pérdida visual.

Sin conflicto de intereses.

\section{BIBLIOGRAFÍA}

1. Cron RQ, Davi S, Minoia F, Ravelli A. Clinical features and correct diagnosis of macrophage activation syndrome. Expert Rev Clin Immunol. 2015; 11 (9): 1043-1053. doi: 10.1586/1744666x.2015.1058159.

2. Bracaglia C, Prencipe G, De Benedetti F. Macrophage activation syndrome: different mechanisms leading to a one clinical syndrome. Pediatr Rheumatol Online J. 2017; 15 (1): 5. doi: 10.1186/s12969-016-0130-4.

3. Larroche C, Mouthon L. Pathogenesis of hemophagocytic syndrome (HPS). Autoimmun Rev. 2004; 3 (2): 69-75. doi: 10.1016/S1568-9972(03)00091-0.

4. Henter JI, Horne A, Aricó M et al. HLH-2004: Diagnostic and therapeutic guidelines for hemophagocytic lymphohistiocytosis. Pediatr Blood Cancer. 2007; 48 (2): 124131. doi: 10.1002/pbc.21039.

5. Shabbir M, Lucas J, Lazarchick J, Shirai K. Secondary hemophagocytic syndrome in adults: a case series of 18 patients in a single institution and a review of literature. Hematol Oncol. 2011; 29 (2): 100-106. doi: 10.1002/hon.960.
6. Ravelli A, Grom AA, Behrens EM, Cron RQ. Macrophage activation syndrome as part of systemic juvenile idiopathic arthritis: diagnosis, genetics, pathophysiology and treatment. Genes Immun. 2012; 13 (4): 289-298. doi: 10.1038/ gene.2012.3.

7. Petty RE, Southwood TR, Manners P et al. International league of associations for rheumatology classification of juvenile idiopathic arthritis: second revision, Edmonton, 2001. J Rheumatol. 2004; 31 (2): 390-392.

8. Ravelli A, Minoia F, Davì S et al. 2016 Classification criteria for macrophage activation syndrome complicating systemic juvenile idiopathic arthritis: a European League Against Rheumatism/American College of Rheumatology/Paediatric Rheumatology International Trials Organisation Collaborative Initiative. Arthritis Rheumatol. 2016; 68 (3): 566-576. doi: 10.1002/art.39332.

9. Stéphan JL, Koné-Paut I, Galambrun C, Mouy R, BaderMeunier B, Prieur AM. Reactive haemophagocytic syndrome in children with inflammatory disorders. A retrospective study of 24 patients. Rheumatology (Oxford). 2001; 40 (11): 12851292. doi: $10.1093 /$ rheumatology/40.11.1285.

10. Zeng HS, Xiong XY, Wei YD, Wang HW, Luo XP. Macrophage activation syndrome in 13 children with systemic-onset juvenile idiopathic arthritis. World J Pediatr. 2008; 4 (2): 97101. doi: 10.1007/s12519-008-0018-6.

11. Dubuc CE, Cáceres VA, Ecenarro MU et al. Síndrome de activación macrofágica secundario a enfermedades autoinmunes, hematológicas, infecciosas y oncológicas. Serie de 13 casos clínicos y una revisión bibliográfica. Reumatol Clin. 2015; 11 (3): 139-143. doi: 10.1016/j. reuma.2014.06.007.

12. Chylack LT Jr, Bienfang DC, Bellows AR, Stillman JS. Ocular manifestations of juvenile rheumatoid arthritis. Am J Ophthalmol. 1975; 79 (6): 1026-1033. doi: 10.1016/00029394(75)90689-3.

13. Abdwani R. Challenges of childhood uveitis. Sultan Qaboos Univ Med J. 2009; 9 (3): 247-256.

14. Castagna I, Roszkowska AM, Alessandrello F et al. Juvenile idiopathic arthritis-associated uveitis: a retrospective analysis from a centre of South Italy. Int Ophthalmol. 2020; 40 (2): 335-342. doi: 10.1007/s10792-019-01184-8.

15. Suhr KS, Chiang MF, Flynn JT, Engelbert M. Ocular involvement in hemophagocytic syndrome: a novel funduscopic manifestation and review of the literature. Retin Cases Brief Rep. 2016; 10 (4): 345-348. doi: 10.1097/ ICB.0000000000000255.

16. Vizcaino MA, Eberhart CG, Rodriguez FJ. Hemophagocytic lymphohistiocytosis in adults with intraocular involvement: clinicopathologic features of 3 cases. Ocul Oncol Pathol. 2017; 4 (1): 1-11. doi: 10.1159/000475551.

17. Ruscitti $P$, Rago $C$, Breda $L$ et al. Macrophage activation syndrome in Still's disease: analysis of clinical characteristics and survival in paediatric and adult patients. Clin Rheumatol. 2017; 36 (12): 2839-2845. doi: 10.1007/s10067-017-3830-3.

Dirección para correspondencia:

Dr. Miguel García Domínguez

Hospital Pediátrico de Sinaloa.

Blvd. Constitución s/n,

Col. Almada, Culiacán,

Sinaloa, México.

Teléfono: 713-35-23 ext 247

E-mail: miguelgarcia.alergia@gmail.com 